\title{
Une approche lagrangienne pour la simulation d'interactions particule/particule en écoulement
}

\author{
par Alain Berlemont, Zhezou Chang, Gérard Gouesbet
}

du Laboratoire d'Energétique des Systèmes et Procédés, de l'URA-CNRS de Rouen

\section{INTRODUCTION}

Les écoulements diphasiques jouent un rôle important dans de nombreuses situations naturelles ou industrielles d'aspects très différents. Ainsi, si des concentrations très faibles peuvent être rencontrées par exemple dans les systèmes de filtration des chambres propres, à l'inverse les éruptions volcaniques génèrent des nuages hautement chargés en particules. Dans l'environnement, on peut être confronté au problème du transport de polluants dans l'atmosphère à faible concentration, mais aussi à la formation de flocons de neige par agglomération de gouttelettes d'eau autour d'un noyau initial, dans un nuage à forte concentration. Dans les procédés industriels, les écoulements peuvent être fortement turbulents et très chargés en particules, comme par exemple les moteurs de propulseurs à combustible solide, les cyclones, la génération et le transport de sprays, ou presque au repos, mais avec de fortes concentrations comme dans les problèmes de sédimentation ou encore les transports de sédiments dans les écoulements fluviaux ou maritimes.

La modélisation du comportement de dispersions particulaires dépend de nombreux paramètres liés aux caractéristiques de l'écoulement porteur (taux de turbulence plus ou moins importants, échelles caractéristiques plus ou moins grandes, géométries plus ou moins perturbées, parois) ou aux particules elles-mêmes (temps de réponse, diamètre, concentration massique, concentration volumique, évaporation, combustion). La complexité des phénomènes à prendre en compte suivant la valeur de ces différents paramètres nécessite un travail de recherche fondamentale important, et chaque étape dans le développement des codes de calcul doit être validée, dans la mesure où des résultats expérimentaux sont disponibles, sur des situations réelles.

Dans les situations les plus simples, il s'agit de caractériser la dispersion de particules dans un écoulement donné en considérant les particules conme un contaminant passif. Mais quand la charge de la phase discrète augmente, la modification de l'écoulement porteur par les particules doit être prise en compte (two-way coupling): ainsi la turbulence contrôle le mouvement particulaire et en retour les particules modifient les caractéristiques de l'écoulement. De nouveaux comportements sont également à considérer lorsque des transferts de masse et de chaleur sont présents (évaporation), ou des réactions chimiques et des transferts radiatifs (combustion) [1] et [2].

\section{Lagrangian approach for the simulation of particle interactions in two phase flows}

Hydrodynamic interactions between particle pairs are studied following sedimentation theory. For wo spheres, interactions are directly obtained through the resistance matrix which links interaction forces to particle relative velocities. For more than two spheres, the mobility matrix is estimated with a four order approximation on the particle distance, and then it is inversed to get the resistance matrix. A set of motion equations (coupled by the resistance matrix) is then solved to describe the particle behaviours. Results for sedimenting particles and collisions in low turbulence field are presented. 
Même si des recherches restent nécessaires sur ces problèmes, il apparaît indispensable d'évoluer vers des milieux plus denses et de développer des études sur la prise en compte d'interactions particulaires (four-way coupling). Ces interactions peuvent faire intervenir différents processus, à savoir des interactions hydrodynamiques particulièrement importantes dans les problèmes de sédimentation, les collisions particules/particules (élastiques ou inélastique, coalescence...) ou encore la modification locale de l'écoulement porteur, différente de la modification globale du two-way coupling (effet de sillage, désintégration...).

\section{DIFFERENTES APPROCHES}

Les simulations d'écoulements diphasiques peuvent être faites suivant différentes méthodes, tant pour l'écoulement porteur que pour la phase transportée. Ainsi, dans le cas d'écoulements turbulents, le fluide pourra être décrit par un modèle classique de fermeture au premier ordre $(k-\varepsilon)$, ou un modèle de fermeture au second ordre (contraintes de Reynolds), ou un modèle de type Large Eddy Simulation, ou encore suivant une méthode de simulation directe, en évoluant du plus simple au plus sophistiqué. De même pour la phase discrète, une méthode eulérienne (les particules sont considérés comme un continuum) ou une approche lagrangienne (suivi de particules individuelles) sont possibles [3].

Les recherches sur les interactions particule/particule, qui n'en sont encore qu'à leur début, peuvent être développées suivant différentes méthodes qui doivent être à la fois complémentaires et interactives, par exemple:

- une simulation lagrangienne de trajectoires individuelles où les collisions sont simulées suivant une succession de processus aléatoires (probabilité de collision, caractérisation de la particule rencontrée...) [4],

- une simulation lagrangienne de plusieurs trajectoires simultanées et la simulation réelle des collisions [5];

- une approche eulérienne où les collisions sont déterminées par analogie avec la théorie cinétique des gaz [6]

Les recherches que nous présentons ici ont été poursuivies dans le cadre de la seconde approche, à savoir la simulation simultanée d'un ensemble de trajectoires et des chocs éventuels entre deux particules.

\section{INTERACTIONS HYDRODYNAMIQUES}

\subsection{Sédimentation}

Les forces dinteractions hydrodynamiques entre plusieurs sphères proches sont issues de la théorie de la sédimentation. Ces interactions ont été estimées dans le cas d'un fluide au repos, mais les problèmes plus spécifiques des contacts entre sphères ne sont pas traités. Sous une hypothèse de petit nombre de Reynolds, la description de l'écoulement fluide entre des sphères proches, donc en interaction, a été largement étudiée [7], [8], [9]. Une première étape dans notre travail a permis de déterminer les forces d'interactions hydrodynamiques entre deux sphères par le biais de la matrice de résistance dont les éléments sont calculés pour deux sphères identiques. Les résultats obtenus sont en bon accord avec des résultats analytiques et expérimentaux d'autres auteurs [10]. Dans cette première approche, nous avons suivi les travaux de Jeffrey et d'Onishi [11] pour deux sphères en interaction. Une solution complète pour trois sphères ou plus est beaucoup plus complexe que pour deux particules, de part la difficulté à exprimer sous forme analytique la matrice de résistance caractérisant le mouvement particulaire. Cependant, sous des hypothèses justifiées et suivant [12], on peut définir la matrice de mobilité qui par inversion conduit à la matrice de résistance.

Les principales hypothèses sont les suivantes:

- les particules sont solides et sphériques.

- les particules sont en translation (pas de rotation),

- le nombre de Reynolds particulaire est petit devant 1

La matrice de mobilité s'écrit alors:

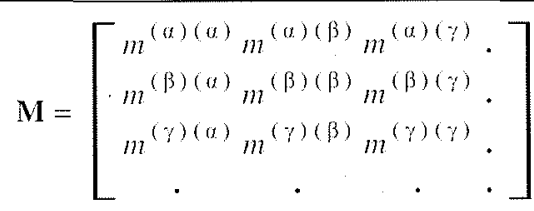

Chaque élément $m^{(\omega)(\beta)}$ de la matrice de mobilité est un tenseur du second ordre attaché aux particules $\alpha$ et $\beta$ :

$$
m_{i j}^{(\alpha)(\beta)}(\vec{r})=x_{\alpha \beta}^{m}(\vec{r}) e_{i} \cdot e_{j}+y_{\alpha \beta \beta}^{m}(\vec{r})\left(\delta_{i j}-e_{i} \cdot e_{j}\right)(2)
$$

où $\vec{e}=\vec{r} / r$ est le vecteur unité entre les centres des deux sphères, $x_{\alpha \beta \beta}^{m}$ and $y_{\alpha \beta}^{m}$ sont des fonctions scalaires qui dépendent des rayons des sphères et de la distance entre les centres, par l'intermédiaire de deux nombres adimensionnels $s=2 r /\left(a_{\alpha}+a_{\beta}\right)$ et $\lambda=a_{0} / a_{\beta}$ (rapport des rayons) qui sont égaux respectivement à $r a$ et 1 dans notre étude (sphères identiques de diamètre $2 a$ ). A partir des résultats de Jeffrey \& Onishi [11] et à l'aide d'un logiciel de calcul formel (MAPLE) on obtient des relations algébriques pour $x_{\alpha, \beta}^{m}$ and $y_{\alpha \beta}^{m}$ comme somme de puissances de $s$ et $\lambda$.

Suivant [12], une approximation au quatrième ordre donne :

$$
\begin{aligned}
& x_{\alpha u}^{m} \quad=x_{\beta \beta}^{m}=1 \quad y_{\alpha u}^{m} \quad=y_{\beta \beta}^{m}=1 \\
& x_{\alpha \beta}^{m}=x_{\alpha \beta}^{m}=\frac{3}{2} s^{-1}-s^{-3} \text { et } y_{\alpha \beta}^{m}=y_{\beta \alpha}^{m}=\frac{3}{4} s^{-1}+\frac{1}{2} s^{-3} \\
& \text { On obtient alors } m_{11}^{(\alpha)(\alpha)}=m_{22}^{(\alpha)(\alpha)}=1 \text { et } \\
& m_{12}^{(\alpha)(\alpha)}=m_{21}^{(\alpha)(\alpha)}=0 \text {. }
\end{aligned}
$$

On peut ainsi déterminer la matrice de mobilité qui est symétrique, définie positive et son inverse $\mathbf{R}$ (matrice de résistance) est calculée par la méthode de factorisation de Cholesky.

En supposant que le terme de Basset, le terme de masse ajoutée et le terme de gradient de pression peuvent être négligés dans l'équation du mouvement des particules, on obtient un système d'équations pour les $n$ particules suivies: 


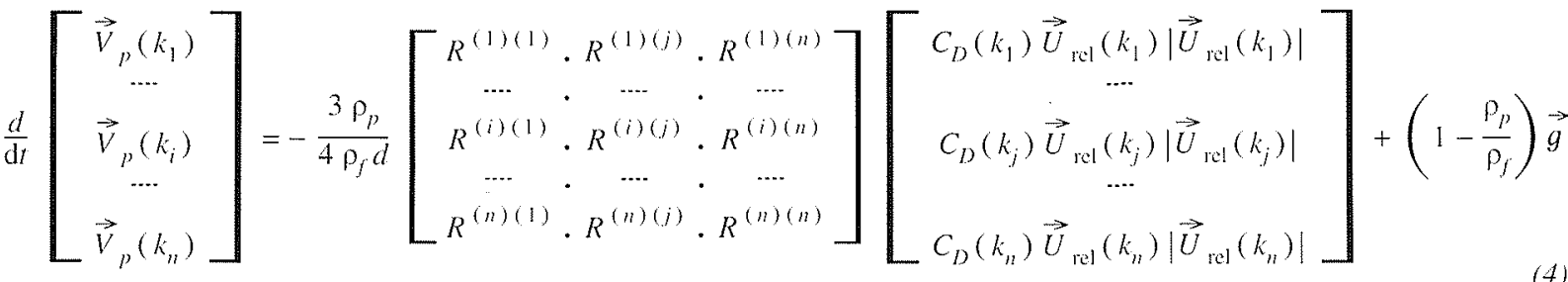

$\vec{V}_{p}(0)$ est la vitesse de la particule $x$.

- $\vec{U}(\vec{x}(\alpha))$ est la vitesse du fluide à la position $\vec{x}(\alpha)$.

$C_{D}(\alpha)=24 / \operatorname{Re}_{p}(\alpha)$

$\operatorname{Re}_{p}(\alpha)=\left|\vec{U}_{\mathrm{rel}}(\alpha)\right| \cdot d / \mathrm{v}$ et

$\vec{U}_{\text {rel }}(\alpha)=\vec{V}_{p}(\alpha)-\vec{U}(\vec{x}(\alpha))$

Une méthode de Runge-Kutta d'ordre quatre permet de résoudre le système où la matrice de résistance est estimée à chaque étape du calcul, c'est-à-dire après chaque estimation des nouvelles vitesses particulaires. On notera que la rotation des particules qui est négligée existe cependant physiquement mais elle est de l'ordre de $\mathrm{O}\left(\mathrm{s}^{-4}\right)$ et s'avère négligeable dans les cas étudiés [8]

A titre d'exemple, nous avons simulé le comportement de quatre sphères (billes d'acier de $1 \mathrm{~mm}$ de diamètre dans de l'huile de silicone) situées initialement dans un plan vertical suivant la géométrie de la figure /. Les trajectoires

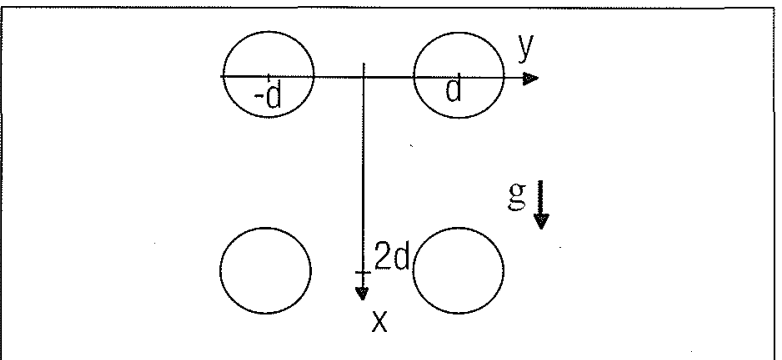

\section{Position initiale des quatre sphères.}

des quatre sphères sont présentées sur la figure 2. Un comportement pseudopériodique est observé, symétrique par rapport à $y=0$. La figure 3 permet de visualiser sur une demi-période le comportement très intéressant des particules. Les deux particules de «devant» s'écartent sous l'effet de forces de pression exercées par les deux de derrière, qui elles mêmes se rapprochent l'une de l'autre. Les deux particules de derrière passent entre les deux de devant, avant que le mouvement inverse ne se produise. Les quatre particules reforment ensuite un carré, les sphères de devant étant alors derrière et inversement. Le même processus continue et conduit au comportement de la figure 2 .

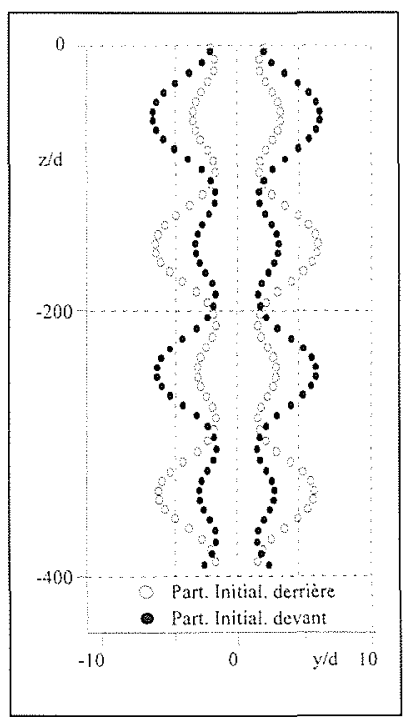

2. Sédimentation de quatre sphères.

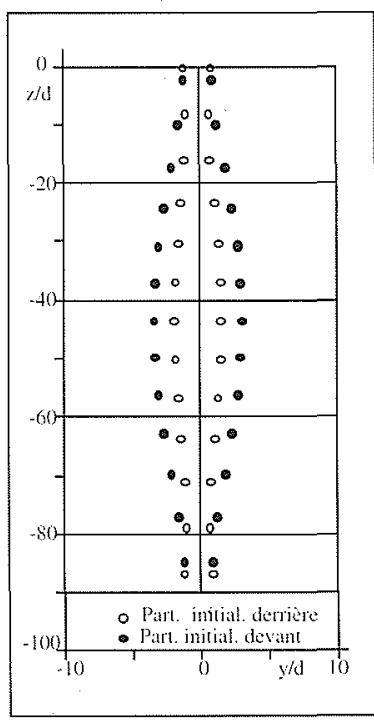

3. Inversion des positions.
Des simulations pour trois sphères donnent également des comportements intéressants qui ont stimulé une étude sur un montage expérimental très simple. Un bon accord est obtenu entre les simulations et les résultats expérimentaux, plus qualitatifs que quantitatifs.

\subsection{Collisions particulaires}

Seules les collisions binaires sont considérées. Quand deux particules $\alpha$ et $\beta$ entrent en collision, c'est-à-dire quand la distance entre les centres des sphères (supposées de même diamètre) est plus petite que le diamètre des particules, on effectue un changement de coordonnées représenté sur la figure 4.

A partir du système de coordonnées de référence $\left(e_{i}\right)$ on définit un nouveau système $\left(e_{i}^{\prime}\right)$ dans lequel le premier vecteur est le vecteur unité dans la direction des centres des sphères.

L'indice „désignant des vitesses après collision, on a :

$$
\begin{aligned}
& \left(V_{\alpha 1}^{\prime}\right)_{a c}=\left[(1-k) V_{\alpha 1}^{\prime}+(1+k) V_{\beta 1}^{\prime}\right] / 2 \\
& \left(V_{\beta 1}^{\prime}\right)_{a c}=\left[(1+k) V_{\alpha 1}^{\prime}+(1-k) V_{\beta 1}^{\prime}\right] / 2 \\
& \left(V_{\alpha, 2}^{\prime}\right)_{a c}=V_{\alpha 2}^{\prime}\left(V_{\beta 2}^{\prime}\right)_{a c}=V_{\beta 2}^{\prime}
\end{aligned}
$$




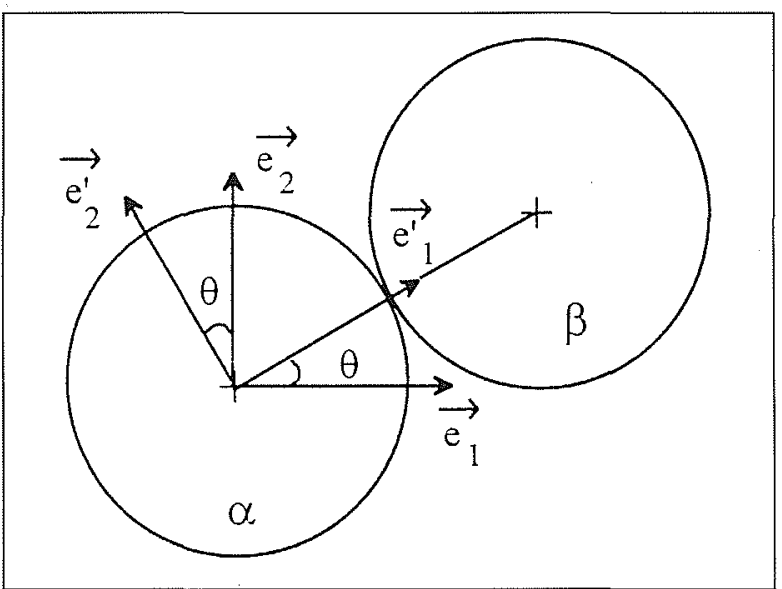

4. Collision : changement de repère.

où $k$ est le coefficient de restitution. La rotation éventuelle des particules n'est pas prise en compte. Un changement de coordonnées inverse nous conduit alors aux vitesses dans le référentiel de travail. Cette procédure est introduite dans la simulation lagrangienne du comportement de particules discrètes décrite ci-dessous.

\section{SIMULATION LAGRANGIENNE}

L'approche lagrangienne que nous développons repose sur la simulation simultanée de deux trajectoires distinctes, l'une pour le fluide et la seconde pour la particule discrète [1], [3].

\subsection{Particule fluide}

Le problème essentiel pour suivre une particule fluide est la détermination de la partie fluctuante de sa vitesse. Suivant une méthode classique dans les approches lagrangiennes, nous utilisons un processus aléatoire qui suit une p.d.f. gaussienne mais aussi respecte les corrélations lagrangiennes de vitesse. Dans les méthodes classiques, le processus utilisé impose la forme de la corrélation. Notre méthode permet, à l'aide d'une matrice de corrélation, de choisir a priori cette corrélation et de conditionner le processus aléatoire suivant cette corrélation. Nous utilisons dans nos calculs une corrélation exp-cos [13] qui contient un paramètre permettant de générer des boucles négatives dans les corrélations. Mais il est cependant important de noter que les paramètres essentiels dans ces processus sont les échelles de turbulence, la dispersion particulaire étant très sensible à ces échelles.

\subsection{Particule discrète}

La trajectographie des particules discrètes consiste principalement à estimer les forces agissant sur la particule et à résoudre ensuite l'équation fondamentale de la dynamique. Pour intégrer cette équation, on doit connaître la vitesse instantanée du fluide au point où se trouve la particule discrète. Pour cela, on suit simultanément une particule fluide et une particule discrète, qui sont initia- lement au même point. La trajectoire de la particule fluide est construite grâce à la méthode exposée précédemment. mais le problème est de déterminer la valeur de la fluctuation de vitesse du fluide, au point $P$ où se trouve la particule discrète. La fluctuation de vitesse au point $P$ est déduite de la fluctuation au point $F$ où se trouve la particule fluide par l'intermédiaire de corrélations spatiales eulériennes.

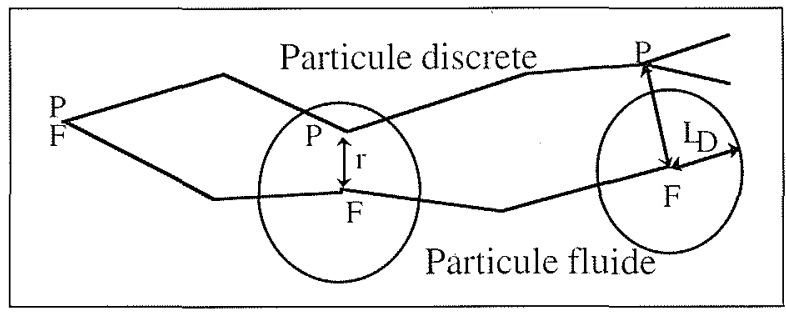

5. Méthode de trajectographie.

Cependant, la corrélation entre les vitesses aux points $F$ et $P$ doit être significative, c'est-à-dire les points $F$ et $P$ doivent être suffisamment proches l'un de l'autre. On définit pour cela un domaine de corrélation autour de la particule fluide à l'aide d'une échelle de longueur $L_{D}$; quand la particule discrète sort de ce domaine de corrélation, on lui associe alors une nouvelle particule fluide, que l'on suit à partir du point $\mathrm{P}$ où se trouve la particule discrète.

Ce processus permet de représenter physiquement l'effet de croisement de trajectoire. $L_{D}$ est définie à partir des échelles spatiales eulériennes de la turbulence.

\subsection{Influence des particules sur la turbulence}

Dans la plupart des situations que l'on rencontre, le débit massique de la phase dispersée n'est pas négligeable devant le débit massique de l'écoulement porteur, et l'influence des particules sur la structure de l'écoulement doit être prise en compte. Une méthode itérative permet de calculer les termes d'échange de quantité de mouvement et d'énergie entre ces deux phases. L'écoulement porteur étant décrit par un modèle de turbulence en $k-$, l'action des particules sur la turbulence intervient par l'intermédiaire de termes de sources introduits dans l'équation de quantité de mouvement, et dans les équations pour l'énergie cinétique de turbulence et sa dissipation. Si, de plus, un changement de phase intervient au niveau des particules, pour des gouttes s'évaporant par exemple, le changement de phase modifie l'influence de la phase dispersée sur l'écoulement porteur. D'une part, la vapeur libérée par les gouttes est une source de masse pour le fluide et, d'autre part, l'évaporation modifie les transferts de quantité de mouvement et d'énergie entre les deux phases. Ce couplage a donné lieu à de nombreuses validations, en particulier dans le cas de jets turbulents. 


\section{PRINCIPAUX RÉSULTATS}

\subsection{Influence de la turbulence}

Les forces d'interactions hydrodynamiques entre les particules ont ensuite été introduites dans un écoulement turbulent homogène et isotrope. Les effets des interactions ont tout d'abord été testés sur un alignement régulier de 10 sphères. Différents paramètres interviennent alors sur l'effet des forces d'interactions: l'intensité de la turbulence, le rayon du domaine d'interaction, la distance initiale entre sphères, et la direction initiale, verticale ou horizontale de l'alignement. Le taux de turbulence initial de $3 \%$ n'a pas permis d'observer une influence des interactions hydrodynamiques. Seuls les chocs, bien que traités simplement (parfaitement élastiques) ont apporté une modification du comportement des particules. Le taux de turbulence a alors été divisé par trois, puis par dix, ce qui a permis d'observer l'effet des interactions hydrodynamiques.

L'étude d'un nuage de 50 particules, dont les positions initiales sont tirées de façon aléatoire mais respectant une concentration donnée, permet de mettre en évidence les effets des forces d'interactions et des chocs. Mais cette fois, l'étude porte sur une configuration initiale qui n'est plus symétrique et particulière comme pour l'alignement de 10 sphères et offre des possibilités d'extension à des situations plus proches de la réalité.

II apparaît au vu des premiers résultats obtenus, que les forces d'interactions hydrodynamiques restent relativement peu importantes dès que le taux de turbulence devient plus grand que quelques pour-cent, cela étant également conditionné par la distance moyenne entre les particules. L'influence des collisions est inverse suivant la direction de l'alignement des particules, à savoir par exemple une augmentation des fluctuations de vitesse dans la direction inter-centre particulaire, et une diminution dans la direction perpendiculaire.

Pour un nuage distribué aléatoirement dans une turbulence isotrope, aucune direction n'est privilégiée et les résultats ne montrent pas de variations sensibles des fluctuations de vitesses dans les cas étudiés. Par contre il semble que pour un écoulement anisotrope, les collisions aient pour premier résultat de provoquer un retour vers l'isotropie des particules.

\section{VI ÉTUDE SPÉCIFIQUE DES COLLI- SIONS}

\subsection{Description de l'écoulement}

L'écoulement porteur est 2D, vertical descendant, avec:

Vitesse moyenne: $U_{f 1}=6.55 \mathrm{~ms}^{-1} ; U_{f 2}=0 . \mathrm{ms}^{-1}$

Echelle intégrale Lagrangienne : $\tau_{L}=0.091 \mathrm{~s}$.

Echelle de longueur Eulérienne: $L_{E ! 1}=0.012 \mathrm{~m}$; $L_{E 11}=0.006 \mathrm{~m}$

L' intensité de turbulence est précisée suivant les cas présentés.

\subsection{Particules}

Des particules de verre de diamètre 70,100 ou $140 \mu \mathrm{m}$ sont injectées dans cet écoulement avec des vitesses initiales moyennes et fluctuantes égales à celles du fluide.

Nous avons simulé 5000 lancers de 50 particules simultanées distribuées initialement de manière aléatoire.

\subsection{Turbulence isotrope}

On a fixé l'intensité de turbulence à $1,2 \%$ c'est-à-dire $\bar{u}_{f i}^{2}=57.2 \mathrm{~cm}^{2} \mathrm{~s}^{-2}$. La distance moyenne initiale entre les particules est de 2.8 diamètres.

On constate sur les figures 6 et 7 que les collisions ne modifient pas les fluctuations de vitesses des particules. Cela s'explique par le fait que les collisions ont une influence inverse suivant la ligne des centres des particules. Or les particules sont initialement distribuées aléatoirement et aucune direction n'est privilégiée. L'augmentation et la diminution des fluctuations de vitesses s'équilibrent.

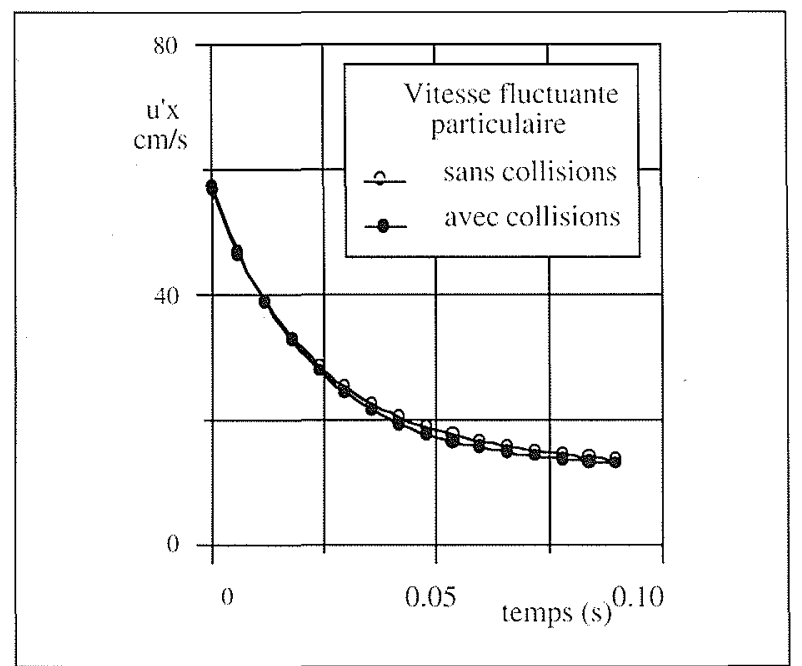

6. Fluctuation de vitesse longitudinale en turbulence isotrope.

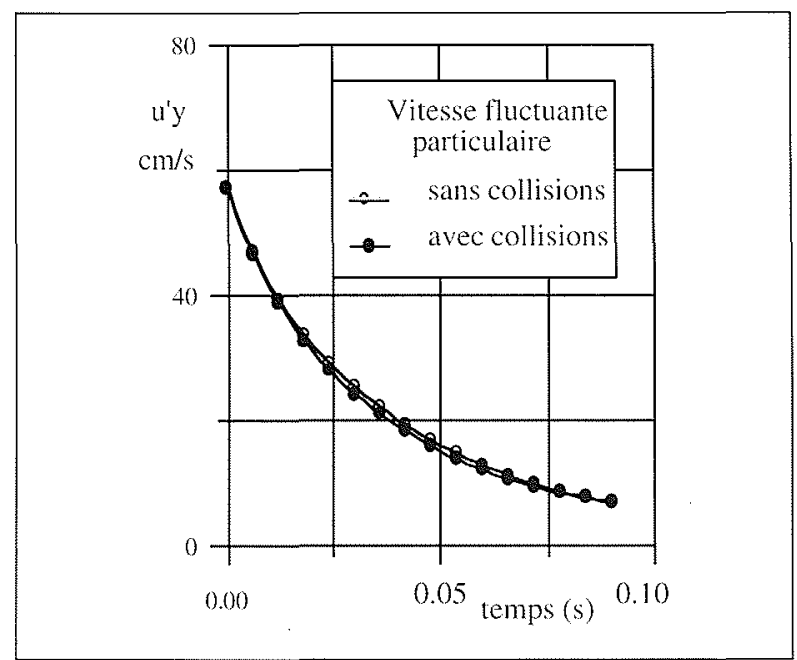

7. Fluctuation de vitesse transversale en turbulence isotrope. 


\subsection{Turbulence anisotrope}

Les conditions de simulations précédentes sont conservées, mais une anisotropie est introduite sur l'écoulement porteur, à savoir :

$$
\bar{u}_{f x}^{2}=57.2 \mathrm{~cm}^{2} \mathrm{~s}^{-2} \text { et } \quad \bar{u}_{j y}^{2}=19.2 \mathrm{~cm}^{2} \mathrm{~s}^{-2}
$$

Les résultats obtenus pour des particules de $100 \mu \mathrm{m}$ sont présentés sur la figure 8 . On constate pour les temps courts $(<0.08 \mathrm{~s})$ une diminution des fluctuations longitudinales et une augmentation des fluctuations transversales, que l'on peut interpréter comme un «retour vers l'isotropie du mouvement particulaire ». Remarquons qu au fur et à mesure que le temps augmente, l'effet devient plus faible du fait de la dispersion turbulente qui produit une nette décroissance des chocs particulaires (fig. 9).

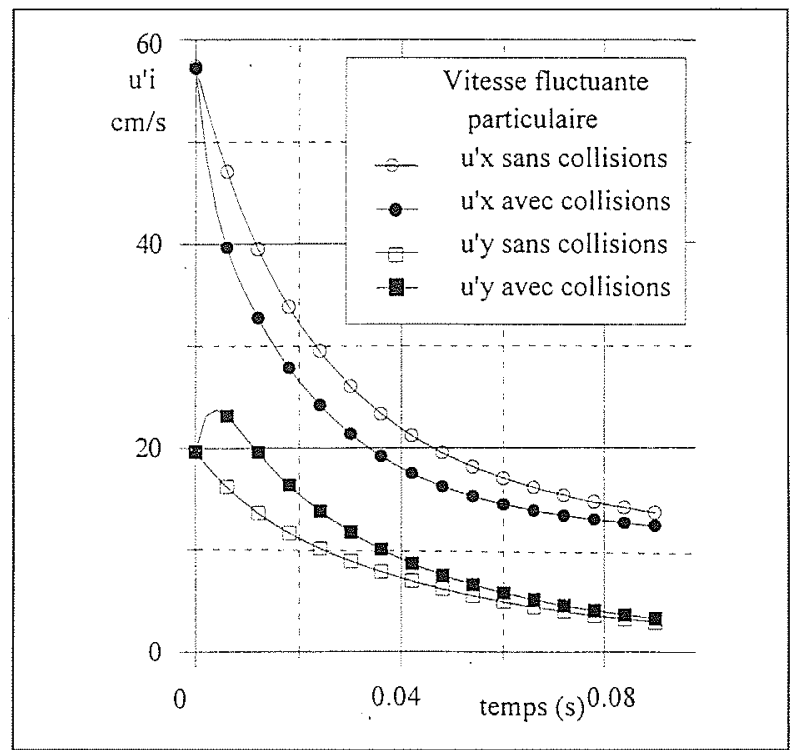

8. Fluctuation de vitesse en turbulence anisotrope.

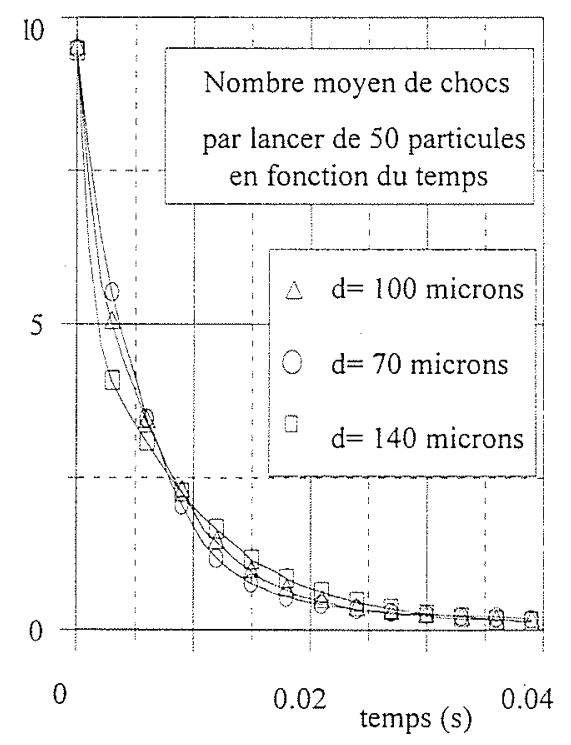

9. Nombre de chocs en turbulence anisotrope.

\subsection{Autres cas étudiés}

Nous avons également étudié lors de cette étude l'influence du temps de réponse particulaire, de l'échelle intégrale lagrangienne et du taux de turbulence. Ces résultats peuvent être trouvés dans la référence [5].

\section{CONCLUSIONS ET PERSPECTIVES}

Les résultats prometteurs obtenus ne sont que le début de l'étude d'écoulements diphasiques turbulents denses. Notamment, ils ne prennent pas en compte les effets de sillage turbulent des particules pour des nombres de Reynolds plus grands que 1. De plus, l'application du code à des particules non solides, comme par exemple des gouttes, implique une approche différente pour le traitement des chocs. Les collisions doivent être alors traitées plus spécifiquement, avec notamment les problèmes de coalescence et de déformation des particules dans le cas de gouttes.

Il apparaît clairement qu'une étape importante pour la suite de ce travail est la validation par comparaisons de nos simulations en écoulement turbulent avec des résultats expérimentaux ou numériques. Les résultats expérimentaux, ou d'autres simulations numériques, permettant une comparaison efficace, commencent à être disponibles dans la littérature et surtout dans les différents laboratoires avec lesquels nous sommes en relation, et vont donc permettre de poursuivre le développement de cet axe de recherche.

De plus il pourrait s'avérer bénéfique d'étudier l'influence de l'isotropisation (généré par les collisions) du mouvement particulaire sur la turbulence, « influence du four-way coupling sur le two-way coupling ».

Ces travaux préliminaires nous permettront d'évoluer vers l'évaporation de nuage de gouttes prenant en compte le couplage entre les gouttes suite à la modification dans l'écoulement porteur des différents produits de vaporisation obtenus (interactions production, diffusion, convection). Nous souhaitons ainsi pouvoir déterminer une cartographie des fractions massiques de chaque constituant dont la prise en compte est indispensable au suivi thermodynamique de la goutte. De plus, il sera nécessaire de coupler à ces interactions les collisions éventuelles entre gouttes, donc d'étudier les problèmes de coalescence en présence d'évaporation.

\section{Références}

[1] Berlemont A., Desjonqueres P. and Gouesbet G. (1990). - Particle lagrangian simulation in turbulent flows, Int. $\mathrm{J}$. of Multiphase Flows, Vol. 16, No 1, pp. 19-34.

[2] Berlemont A., Desjonqueres P, and Gouesbet G. (1993). - Particle tracking in turbulent flows. Invited paper, ASME Gas-Solid Flows Conference, Washington D.C. 20-24 June, Proceedings FED-Vol. 166, pp. 121-131.

[3] Berlemont A., Grancher M.S. and Gouesbet G. (1991). - On the lagrangian simulation of arbulence infuence on droplet evaporation, Int. J. Heat \& Mass Transfer, Vol. 34 , 11, pp. 2805-2812.

14] SOMMERFELD M. (1995). - The importance of interparticle collisions in horizonfal gas-solid channel flows. ASME/JSME Int. Conf. on Gas-Solid Flows, Hilton Head, South Carolina, USA, FED-Vol. 228, pp. 335-346. 
[5] Berlemont A., BenOIST F. and GOUESBET G. - Influence of collisions on particle fluctuating velocities using a Lagrangian approach. Int. Symp. Num. Meth. for Multiphase Flows, ASME Summer Conf., Lake Tahoe, USA, FEDVol. 185, pp. 23-28. Juin 1994

16] LAvieville J., DeuTsch E. and SMMONin O. (1995). Large Eddy Simulation of interactions between colliding particles in Homogeneous isorropic turbulence field, ASME/JSME Int. Conf, on Gas-Solid Flows, Hilton Head, South Carolina, USA, 13-18 August, FED Vol. 228, pp. 347-358.

[7] STIMSON M. and JEFFerY G.B. (1926). - The motion of two spheres in a viscous fluid. Proc. Roy. Soc., A, Vol. 11 , pp. $110-116$.

[8] HOCKNG L.M. (1964). - The behaviou of clusters falling in a viscous fluid. I. Fluid Mech., 20, pp. 129-139.

[9] Ganatos P., Prefrer R., Weinbaum S. (1978). - A numerical solution rechnique for three dimensional Stokes flows, with applicationto the motion of shongly interacting spheres in a plane. J. Fluid Mech. vol 84, part 1, pp. 79-111.

[10] Chauvin C., Gouesbet G, and Berlemont A. (1993). Influence of particle interactions on particle dispersion in low tubulence field, 2nd Int. Symp. on Eng. Turb. Mod. and Meas., Florence 31 May-2 June, Italy, Proceedings pp. 915925. Rodi W. \& Martelli F. Ed., Elsevier Pub. Co.

[11] JEFFREY D.J. and ONISHI Y. (1984). - The forces and couples acting on wo nealy fouching spheres in low Reynolds number flows, J. Fluid Mech., Vol. 139, pp. $261-290$.

[12] DURlofsky L.F., Brady J.F. and Bossis G. (1987). Dynamic simulation of hydrodynamically imeracting particles, J. Fluid Mech. Vol. 80, pp. 2 [-49.

[13] Frenkiel, F.N. (1948). - Etude Statistique de la Turbulence-Fonctions spectrales et Coefficients de Corrélation, Rapport Technique, ONERA No 34. 